\title{
Spinal Canal Decompression for Stage III Kummell's Disease With "Compressing the Posterior Wall of the Vertebral Body" Guided by Intraoperative Ultrasonography - a Preliminary Clinical Study
}

\section{Guangqing Cao}

The Second Hospital, Cheeloo College of Medicine, Shandong University https://orcid.org/0000-0001-9755-3071

Jidong Zhang

Dongda hospital of Shanxian

\section{Cheng Zhang}

The Second Hospital, Cheeloo College of Medicine, Shandong University

\section{Howard J Ginsberg}

University of Toronto

\section{Manuel Fuetsch}

University of Toronto

\section{Jie Zhao}

The Second Hospital, Cheeloo College of Medicine, Shandong University

\section{Chunzheng Gao}

The Second Hospital, Cheeloo College of Medicine, Shandong University

Dongjin Wu ( $15153169657 @ 163.0 m$ )

The Second Hospital, Cheeloo College of Medicine, Shandong University

\section{Research article}

Keywords: intraoperative ultrasonography, stage III Kummell's disease, compressing the posterior wall of vertebral body decompression

Posted Date: January 20th, 2021

DOI: https://doi.org/10.21203/rs.3.rs-149539/v1

License: (c) (i) This work is licensed under a Creative Commons Attribution 4.0 International License. Read Full License 


\section{Abstract}

Background: There is a lot of controversy regarding the treatment of patients with stage III Kummell's disease (KD). The purpose of this study is to determine feasibility, efficacy and safety of spinal canal decompression via "compressing the posterior wall of the vertebral body", guided by real time intraoperative ultrasonography guidance for patients with stage III $\mathrm{KD}$.

Methods: We conducted a retrospective study of 11 patients with stage III KD associated with neurological deficits who underwent surgical treatment in The Second Hospital, Cheeloo College of Medicine, Shandong University, from September 2017 to January 2019. All patients underwent spinal canal decompression using a special L-shaped compressor (angular compressor) guided by real time ultrasonography to squeeze and compress the posterior wall of vertebral body, supplemented by internal fixation and posterior fusion. The primary goal was to determine feasibility, safety and efficacy. Therefore, operating time, Intraoperative blood loss, complications and JOA score, local Cobb angle, ODI score of pre- and post-operative were evaluated.

Results: 11 patients ( 4 males and 7 females) with an average age of 70.2 \pm 3.2 years (63-76 years) were included in the study. Mean follow-up period was 17.4 months (12-24 months). Mean operating time was $151 \pm 18$ min and mean intraoperative blood loss was $202 \pm 40 \mathrm{ml}$. Before and after surgery, the local Cobb angle ( $34.3^{\circ} \pm 5.4^{\circ}, 11.0^{\circ} \pm 4.6^{\circ}$, respectively), JOA score $(12.8 \pm 2.1,22.1 \pm 1.9$, respectively) and ODI scores $(71.4 \pm 6.4,25.5 \pm 5.2$, respectively) significantly improved $(P<0.05)$. As a complication, one patient developed CSF leakage after surgery, while in another case transient MEP signal change occurred intraoperatively without neurological deficit after surgery.

Conclusion: Spinal canal decompression by tamping the retropulsed vertebral body anteriorly, guided by intraoperative ultrasonography can effectively relieve neural compression, significantly shorten operation time, reduce intraoperative blood loss, and have fewer complications for patients with stage III KD, compared with previously reported data. In our opinion, it therefore represents a low risk, minimally invasive, yet effective treatment strategy in this selected group of patients.

Trial registration: This study was retrospectively registered in Chinese Clinical Trial Registry.

Unique Identifying number: ChiCTR2000040490.

\section{Introduction}

KD was first described by German surgeon Hermann Kummell in the 1890s. It represents a specific type of osteoporotic compression fracture with or without minor trauma, which occurs most frequently in the thoracolumbar vertebrae. ${ }^{1}$ Patients mainly complain about local back pain and tenderness at the injury site. As the disease progresses, kyphosis of the involved segments, potentially followed by neural compression may occur².

Li et $\mathrm{al}^{3}$ divided KD into three stages according to clinical characteristics: stage $\mathrm{I}$, the loss of vertebral body height is less than $20 \%$, with no adjacent disc degeneration, and patients mainly complain lower back pain; stage II, the loss of vertebral body height is more than $20 \%$, usually accompanied by adjacent disc degeneration and/or instability of the fractured vertebral body. These patients primarily present with radiculopathy; stage III, the collapsed posterior wall of vertebral body is displaced dorsally and protrudes into the spinal canal. The patients typically present with lower back pain, often accompanied by severe neurological deficits.

For patients with stage I and II KD, PKP or PVP procedure are usually recommended ${ }^{4-7}$. However, there is a lot of controversy regarding the treatment of patients with stage III KD. In recent years, many authors have reported different treatment options. These surgical methods mainly differ in their approach, including anterior or posterior decompression alone, or a combination. Additionally, anterior and middle column fixation as well as spinal canal decompression can be achieved via the anterior approach. However, in patients with osteoporosis, the stability of anterior column fixation is weaker than that of 
posterior pedicle screw fixation, and anterior surgery is more invasive and with a higher risk of internal organ inujury ${ }^{8}$. Hence, decompression and fusion via a posterior approach has become the treatment of choice for patients suffering from stage III $\mathrm{KD}^{9}$. Historically, surgery via a posterior approach also has its shortcomings: due to the fractures being old and the posterior wall of the vertebral body displacing into the spinal canal, satisfactory decompression of the spinal canal is more difficult. Some scholars have adopted bilateral pedicle entry to remove the displaced vertebrae, followed by the application of bone graft to support the anterior and middle columns. This method can obtain complete spinal decompression ${ }^{2}$. However, the vertebral body and posterior structure of the lesion vertebrae are seriously destroyed in the process, compromising the structural stability of the spine. In addition, slow fusion of the bone due to the nature of osteoporosis often results in internal fixation failure. Meanwhile, this strategy is more invasive for the patient with more blood loss, longer surgery time, and more complications, making the procedure very controversial ${ }^{2}$.

By considering the advantages and disadvantages of current surgical methods for stage III KD, our study combined intraoperative real time ultrasonography guidance with neurophysiology monitoring, avoiding neural elements irritation during anterior displacement of the posterior wall to the ventral side of the spinal canal with a special L-shaped compressor (Trauson, China). Additionally, we also corrected obvious residual kyphosis with a Smith-Peterson osteotomy (SPO) or Ponte osteotomy to achieve better decompression and corrective results in selected patients. Ultrasound provides protection of the neural elements and immediate feedback of successful decompression.

\section{Materials And Methods}

This study was approved by the Ethics Committee of our hospital and conducted according to the principles of the Declaration of Helsinki (Ethical principles for Medical Researches Involving Human Subjects).

We retrospectively collected data on patients with neurological deficits suffering from stage III KD who underwent surgical treatment at our hospital from September 2017 to January 2019.

Inclusion criteria were as follows: stage III KD diagnosed by imaging; neural compression due to the retropulsed posterior wall of the collapsed vertebral body rather than degenerative disc; bone mineral density examination suggesting severe osteoporosis (Table 1).

Exclusion criteria: patients who unfit for surgery; tumors, infections and other factors leading to pathological vertebral fractures, younger than 60 years old.

\section{Surgical approach}

All surgeries were performed by the same surgical team to reduce variability related to surgical techniques.

Under general anesthesia, the patient was placed in the prone position with abdomen suspended and connected to neurophysiology monitor. Adjusting the patient's posture to reduce the fracture by extending the involved levels, and the degree of reduction and the remaining kyphosis was evaluated radiographically with a C-arm.

A posterior midline incision was performed, then the lamina and bilateral facet joints were exposed. Pedicle screws (Medtronic, America) were implanted in two vertebrae above and below the affected level respectively, augmented by bone cement (1.5 ml/trajectory) (Tecres, Italy). Ultrasonic bone blade was used to excise the lamina and the facet joints of the side ( with more severe anterior compression) to facilitate placing the angular compressor to lateral-ventral side of the dural sac in order to minimize retraction of the dura and neural elements as much as possible. The opposite upper and lower facet joints were also resected (Smith-Peterson osteotomy, or Ponte osteotomy if necessary) if residual kyphosis required correction. Bilateral underbent rods were connected to screws to correct kyphosis, which could also contribute indirectly to decompression via shifting of the dural sack posteriorly ${ }^{10,11}$. Appropriate amount of normal saline was added as a medium in the surgical field, and the ultrasonic probe (1202, Brüel \& Kjær, Denmark) was used to detect the sagittal and axial sections 
of the spinal cord, thereby determining the location and degree of compression from the ventral side of the dural sac. The facet joint on the side with more severe symptoms was removed. Then under real-time ultrasonography guidance, the angular compressor was placed ventral to the thecal sac through the safe area on the cephalad or caudal side ${ }^{12}$. Retropulsed bone fragment which compressed neural elements were carefully and gently compacted ventrally. During the procedure, neurophysiology monitoring was closely observed for any signal changes,including SSEP and MEP. After confirming no compression of the neural elements with ultrasonography, both rods were temporarily removed. The cleft in the vertebral body was filled with bone cement via the pedicle of the injured vertebrae, followed by implantation of two polyaxial pedicle screws. The rods were reinserted, and screws properly compressed to correct the kyphotic deformity. Ultrasonography was used again to confirm adequate decompression, and correction confirmed radiographically via C-Arm before tightening the nuts to complete the fixation. Lamina and facet joints of fixed segments were decorticated for bone grafting, autologous bone particles and allogeneic bone chips were used. Drainage was placed and incision was closed in common fashion.

Postoperative antibiotics were used routinely for 24 hours to prevent surgical site infections, supplemented by regular antiosteoporosis treatment; drainage was removed when drainage volume was less than $50 \mathrm{ml} /$ day, and patients were instructed to gradually stand and walk with custom-made braces after 3 days.

Typical routine follows up at our institution include appointments after $3,6,12,18$ and 24 months. Generally,the patients were recommended to take CT scans after 6 and 12 months, and X-rays after 3, 6, 12, 18 and 24 months.

\section{Clinical outcome and statistical analysis}

The patient's preoperative and postoperative JOA score, ODI score, local kyphosis Cobb angle, operation time, intraoperative blood loss, and postoperative complications were recorded in detail.

SPSS 22.0 statistical software was used for analysis. Continuous variables were expressed as $\overline{\mathrm{x}} \pm \mathrm{SD}$. The variance analysis was used for comparison before and after the operation, and the paired sample t test was used for pairwise comparison. $\mathrm{P}<0.05$ was considered statistically significant.

\section{Results}

4 males and 7 females were included in the study. Eight cases had minor trauma. The patients were all accompanied by obvious lower back pain, including 8 patients with numbness and weakness in the lower extremities, 5 patients mainly presented with intermittent claudication, and 2 patients developed perineal numbness and sphincter dysfunction.

The mean age was $70.2 \pm 3.2$ years ( $63-76$ years old). The duration of low back pain was $3.5 \pm 2.3$ months (0.5-12 months). Among them, the distribution of affected segments was 1 case at T11 level, 1 case at T12 level, 6 cases at L1 level, 3 cases at L2 level. The Preoperative Cobb angle was $34.3^{\circ} \pm 5.4^{\circ}$, the preoperative Lumbar JOA score (Japanese Orthopedic Association Scores) $12.8 \pm 2.1$, and the preoperative ODI score was $71.4 \pm 6.4$.

In the present study, mean operative time was $151 \pm 18 \mathrm{~min}(125-180 \mathrm{~min})$ and average blood loss was $202 \pm 40 \mathrm{ml}(150-$ $275 \mathrm{ml})$.

There was no leakage of bone cement into the spinal canal in our case series, and all the pedicle screws were augmented. 4 cases performed Smith-Peterson osteotomy, and 2 cases chose Ponte osteotomy to correct kyphosis.

While no patient showed surgical site infection postoperatively. And one patient suffered from postoperative cerebrospinal fluid leakage. After being laid in Trendelenburg position, and removal of the drain, no obvious symptoms related to low intracranial pressure such as headache appeared, and the incision healed successfully.

While all other intraoperative monitoring results were stable, one patient's MEP amplitude decreased about $40 \%$ during operation, the amplitude gradually recovered after the ventral decompression of the thecal sac, and there was no obvious neurological deterioration after surgery. 
All 11 patients had satisfactory outcomes during follow-up, with a median follow-up time 17.4 months (12-24 months). At the time of final follow-up, the local Cobb angle $\left(34.3^{\circ} \pm 5.4^{\circ}, 11.0^{\circ} \pm 4.6^{\circ}\right.$, respectively), JOA score $(12.8 \pm 2.1,22.1 \pm 1.9$, respectively) and ODI scores $(71.4 \pm 6.4,25.5 \pm 5.2$, respectively) significantly improved compared to the preoperative baseline. $(P<0.05$, Table 1). No patient experienced failure of instrumentation such as screw pulling out or breakage. Additionally, no deterioration of neurological symptoms was recorded.

Table 1

Demographic and clinical details of the 11 patients with stage III Kummell's disease

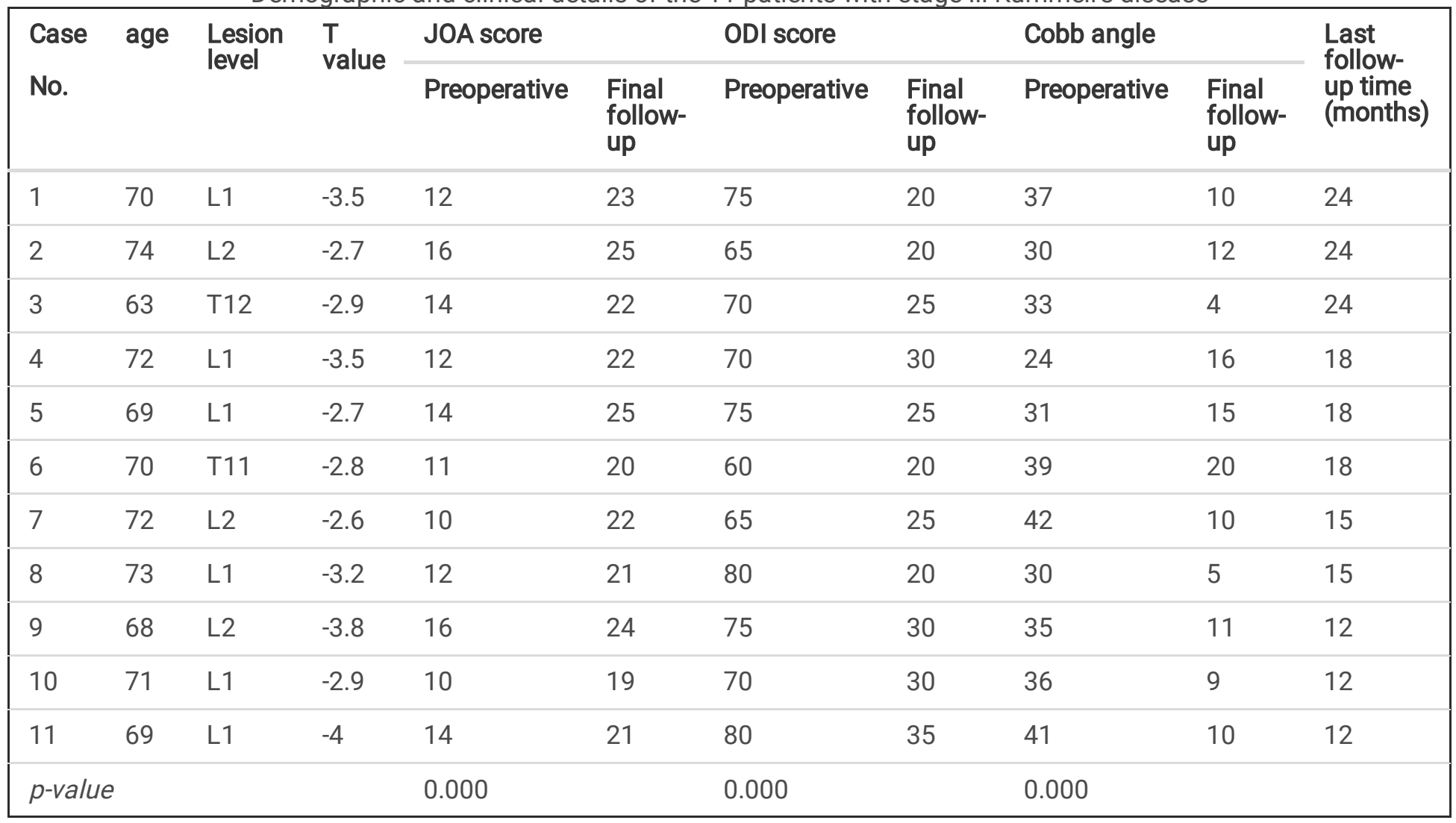

\section{Representative case presentation}

A 72-year-old woman was diagnosed as L1 stage III KD and incomplete paralysis at our hospital. The Patient's lumbar JOA score was 12 points and T value was - 3.5. She was treated with "compressing the posterior wall of the vertebral body" and guided by ultrasonography, combined with vertebroplasty and cement augmented pedicle screw internal fixation.

She recovered uneventfully and was followed up for 2 years. At final follow up, her low back pain and intermittent claudication disappeared. In addition, she only had slight numbness in her feet and walked very well and was pretty satisfied with her surgery.

\section{Discussion}

For patients with stage III KD, due to the compression to the neural elements by the retropulsed posterior wall of vertebral body, PVP or PKP surgery alone may not achieve adequate decompression and relieve neurological symptoms. Hence, open decompressive surgery is often required to achieve satisfactory decompression of the thecal sac and/or nerve roots and correction of local kyphosis ${ }^{3}$. However, current surgical methods of choice are accompanied by a high rate of intra- and perioperative morbidity.

\section{Current surgical methods and their deficiencies}


Previous studies reported that the anterior approach can remove the collapsed and compressed vertebral body and obtain good direct decompression, moreover, stabilization of the anterior and middle columns can be achieved through strut bone grafting ${ }^{13}$. Kanayama et al collected 31 patients who underwent anterior surgical treatment. The mean operative time was $193 \mathrm{~min}$ (150-285 min), the mean intraoperative blood loss $436 \mathrm{ml}$ (100-1350 ml). The mean postoperative follow-up period was 57 months and satisfactory outcome was obtained ${ }^{13}$. However, most of the patients suffering from Stage III KD are elderly. The anterior approach is more invasive and has more intraoperative blood loss and risks internal organ injury. Patients recover slowly and stay bedridden for longer periods. The risks of the anterior approach are relatively greater ${ }^{8,14}$. This suggests that the anterior approach may not be an ideal option for older patients with stage III KD.

The posterior approach is familiar to most spinal surgeons. With the continuous development of posterior surgical techniques and internal fixation instruments, many methods such as osteotomy and corrective maneuvers can be used to accomplish adequate decompression of neural elements deformity correction and obtain rigid spinal stability. However, this requires removal of the vertebral body and the posterior vertebral arch, so the stability of the spine is severely impaired, and the load on internal fixation is greatly increased. Especially for KD patients who usually suffer from serious osteoporosis, the internal fixation failure rate is high ${ }^{15-17}$. Zhang et al ${ }^{2}$ reported 12 cases of chronic spinal cord compression with neurological impairment in stage III KD treated with posterior surgery. The cranial half of the affected vertebral body and the adjacent intervertebral disc were removed through bilateral transpedicular approaches, the anterior and middle columns were reconstructed using a cage. The long segment was fixed. The average operation time of this surgical method is $148 \mathrm{~min}$ (100-220 min), and there was significant bleeding during the operation, with an average of $625 \mathrm{ml}(450-850 \mathrm{ml})$ Furthermore, 2 out of 12 patients presented with neurological deficits postoperatively, questioning the safety of the procedure

\section{Innovations and surgical techniques of this study}

Compression to the thecal sac and the spinal cord in patients with stage III KD derives from a retropulsed posterior wall of the collapsed vertebral body. Furthermore, the retropulsed body fragment is severely osteoporotic, and the posterior wall of the vertebral body is weakened due to the presence of the vertebral basal vein foramen ${ }^{18}$, which makes the retropulsed body fragment easy to be compacted and pushed anteriorly, while the fragment of $\mathrm{DH}$ (Disc herniation) is the soft nucleus pulposus (with a hard bone shell when calcified), and OPLL(Ossification of posterior longitudinal ligament) is a hard cortical bone, which are not easily compacted and compressed ${ }^{19}$. Additionally, compared to compression of a DH or OPLL, the onset of patients with stage III KD is relatively short with no adhesion between the dural sac and the posterior bone fragment. The different characteristics of pathological compression mentioned contribute to the feasibility of the surgical technique presented in this study.

We hereby introduce a new surgical procedure for stage III KD: During the operation, under the real time guidance of ultrasonography, an angular compressor was used to squeeze and compact the posteriorly displaced vertebral body wall and collapse the fragment, which directly decompresses the neural elements and avoids the necessity of a more aggressive approach by corpectomy. Moreover, we used augmented pedicle screw fixation to restore the stability of the spine and bone cement to fill the affected vertebrae to obtain ideal anterior and middle column support. Ultrasonography was used to identify the location of the encroaching fragment from the sagittal plane and the axial plane during the operation ${ }^{20,21}$ and to guide decompression in a real time way to minimize irritation of the dural sac and ensure the safe and complete decompression during the operation. An angular compressor is gently placed between the posterior vertebral body and the ventral side of the dural sac, compact and collapse posterior wall to the vertebral body. The neurophysiological monitoring needed to be closely observed to further ensure the safety of the nerve during the entire operation process. The results showed that Cobb angle, JOA score, and ODI score were significantly improved after surgery, which highlights the efficacy of our procedure.

This technique has obvious advantages compared with the previously reported surgical methods. The posterior approach can avoid interference and reduces complications. Bilateral facet joints can be spared if the local kyphosis is not significant or the lesion is located in the lumbar segment, reducing the damage to the spine's posterior bony structures. The injured vertebral body and upper and lower intervertebral discs were not resected, which could avoid destroying the anterior and middle column 
structure and contribute to preserve the stability of the spine. Meanwhile, the bone cement placed into the injured vertebral body to provide anterior and middle column support, and screws were implanted before the bone cement hardened, so that the bone cement block and internal fixation integrated together to form a single load bearing frame. Therefore, the stability of the spine was restored to the maximum extent which may explain why no internal fixation failure was observed in our study. Additionally, squeezing and compacting the fragment at the posterior vertebral body by the compressor may theoretically close the vertebral basal vein foramen, reducing bone cement leakage into the spinal canal, and improving the safety of the operation ${ }^{22}$. There was no leakage into the spinal canal in our case series, which might illustrate the effectiveness of this technique in reducing leakage of bone cement. Through the application of ultrasound guidance, the location of the surgical instruments and the dura mater could be observed in real time, and compacting the retropulsed posterior vertebral wall could be achieved directly, thoroughly and accurately, minimizing the irritation of neural elements. Only one patient in this group had transient MEP signal changes during intraoperative neurophysiology monitoring, but the patient did not experience neurological deterioration after surgery, and neurological function gradually recovered during follow-up, suggesting the safety and effectiveness of this procedure. In addition, we designed special thin and hard compressor (angular compressor), minimizing encroachment into the spinal canal as much as possible, and it would not be deformed when squeezing and compressing the cancellous bone which also minimized irritating the dural sac. A delicate stripping instrument was employed to release the possible adhesion between dura and bone fragment, so to reduce the risk of a dural tear. Only one case in this group experienced a dural tear, which was lower than the incidence of previous studies ${ }^{23,24}$. Various degrees $\left(90^{\circ}, 120^{\circ}\right.$, etc.) are designed at the tip of the compressor, which was convenient for compressing and compacting the bone fragment. The operative time was relatively shorter, and bleeding and trauma were reduced and fewer neurological complications occurred compared to corpectomy for decompressive surgery. In this study, the average blood loss was $202 \pm 40 \mathrm{ml}(150 \sim 275 \mathrm{ml})$, which was less than that of Zhang et al. ${ }^{2}$ and Yang et al ${ }^{25}$ reported $(625 \mathrm{ml}(450-850 \mathrm{ml}), 860 \pm 130 \mathrm{ml}$, respectively). Jo et al. ${ }^{26}$ reported 13 patients who underwent osteotomy, with an average operation time of 275 minutes and intraoperative bleeding of about $1585 \mathrm{ml}$. One of the patients suffered neurological deterioration due a direct injury to the nerve root during surgery.

From our experience gained during this study, the following are our recommendations for the procedure: Firstly, if there are bridging osteophytes in front of the injured vertebrae with adjacent levels, compression between the pedicle screws of this segment should be avoided to prevent screws loosening. The articular process osteotomy at the other segments was recommended if the obvious kyphosis must be corrected. Secondly, if the residual kyphosis was acceptable or the lesion was located in the lumbar segments, facet joints should not be removed, thus reducing the destruction of the posterior column structure and preserve the bone grafting bed, especially when at the lumbar segments or thoracic ones where ultrasonography indicated the presence of the space between spinal cord and pathological compression when dural sac pulsation before decompression ${ }^{27}$. Thirdly, we recommend performing kyphosis correction first to indirectly release the nerve tissue before decompression, which can improve the safety of surgery 28,29 . Fourth, when putting the compressor to the ventral side of dural sac, we recommend placing it at the adjacent intervertebral disc level first, then add saline for ultrasound visualization. The location of the compressor can be seen under ultrasonography to avoid interfering with the nerve tissue during placement. Fifth, the positional relationship between the nerve tissue and the device must be clearly observed under real time ultrasonography during decompression, and the device must not cause compression to the neural elements. Lastly, the Lshaped angular compressor should first be placed into the cephalic (or caudal) end of the apex of the encroaching bone fragment. After compressing part of the bone fragment, gently and gradually move towards the highest point. To avoid nerve injury, the soft cancellous bone could be compressed first to form a posterior bone culvert, then use a thin osteotome to cut the bone edge of the culvert from both margins outside the dural sac sides, and then press the cortex to the ventral side to complete the decompression.

As mentioned above, our study avoids complications related to anterior surgery approach and severe damage to spinal stability due to corpectomy surgery. Therefore, spinal stability was preserved in all patients, and the rate of instrumentation failure was significantly reduced. All patients in this group achieved bone fusion at last follow-up. Only one case had cerebrospinal fluid leakage, but it did not affect wound healing and neurological recovery. These indicates that this surgical 
procedure was safe and effective in our study population, but a larger number of cases and long-term follow-ups are still needed to confirm our findings.

\section{Limitations of this study}

This study also has certain limitations: the number of cases is small, and the follow-up time for some cases is short, especially considering the late onset of some complications like adjacent segment disease or delayed hardware failure. Because the incidence of stage III KD is relatively low, the analysis results of 11 patients are still valuable. This study is a retrospective study with a therefore impaired level of evidence.

\section{Conclusion}

Spinal canal decompression by "compressing the posterior wall of vertebral body" with intraoperative real time ultrasonography guidance, supplemented with pedicle screw internal fixation and fusion for the treatment of stage III KD, could effectively relieve neural compression and symptoms, significantly shorten operative time, and reduce intraoperative bleeding compared with the previously reported data. We therefore consider it a feasible, effective and safe procedure in patients suffering from fractures of stage III KD.

\section{Abbreviations}

KD: Kummell's disease;

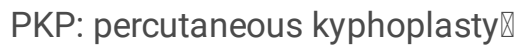

PVP: percutaneous vertebroplasty;

ODIQThe Oswestry Disability Index》

JOA score囚Japanese Orthopedic Association Score;

DH: Disc herniation;

OPLL: Ossification of posterior longitudinal ligament

\section{Declarations}

\section{Ethics approval and consent to participate:}

This study was approved by the Ethics Committee of The Second Hospital, Cheeloo College of Medicine, Shandong University. Reference number: KYLL-2020『LW囚-061.

Trial registration: Chinese Clinical Trial Registry, ChiCTR2000040490. Registered 01 September 2020 - Retrospectively registered, http://www.chictr.org.cn/edit.aspx?pid=64640\&htm=4

\section{Consent for publication:}

Informed consent for publication of photographs and data was obtained from all subjects.

\section{Availability of data and materials:}

The datasets used and/or analyzed during the current study are available from the corresponding author on reasonable request.

\section{Competing interests:}


The authors declare that they have no known competing financial interests or personal relationships that could have appeared to influence the work reported in this paper.

\section{Funding:}

None.

\section{Authors' contributions:}

Study design: Dongjin Wu, Chunzheng Gao

Data collections: Guangqing Cao, Cheng Zhang, Jie Zhao, Jidong Zhang

Date analysis: Guangqing Cao, Jidong Zhang, Dongjin Wu

Writing: Guangqing Cao, Howard J Ginsberg, Manuel Fuetsch, Dongjin Wu

\section{Acknowledgements:}

Not applicable

\section{References}

1. Steel HH. Kummell's disease. Am J Surg 1951;81:161-167.

2. Zhang X, Hu W, Yu J, Wang Z, Wang Y. An Effective Treatment Option for Kummell Disease With Neurological Deficits: Modified Transpedicular Subtraction and Disc Osteotomy Combined With Long-Segment Fixation. Spine (Phila Pa 1976) 2016;41:E923-930.

3. Li KC, Li AF, Hsieh CH, Liao TH, Chen $\mathrm{CH}$. Another option to treat Kummell's disease with cord compression. Eur Spine J 2007;16:1479-1487.

4. Huang Y, Peng M, He S, Tang X, Dai M, Tang C. Clinical Efficacy of Percutaneous Kyphoplasty at the Hyperextension Position for the Treatment of Osteoporotic Kummell Disease. Clin Spine Surg 2016;29:161-166.

5. Clark W, Bird P, Gonski P, et al. Safety and efficacy of vertebroplasty for acute painful osteoporotic fractures (VAPOUR): a multicentre, randomised, double-blind, placebo-controlled trial. Lancet 2016;388:1408-1416.

6. Buchbinder R, Osborne $\mathrm{RH}$, Ebeling PR, et al. A randomized trial of vertebroplasty for painful osteoporotic vertebral fractures. N Engl J Med 2009;361:557-568.

7. Firanescu CE, de Vries J, Lodder P, et al. Vertebroplasty versus sham procedure for painful acute osteoporotic vertebral compression fractures (VERTOS IV): randomised sham controlled clinical trial. BMJ 2018;361:k1551.

8. Mochida J, Toh E, Chiba M, Nishimura K. Treatment of osteoporotic late collapse of a vertebral body of thoracic and lumbar spine. J Spinal Disord 2001;14:393-398.

9. Liu FY, Huo LS, Liu S, et al. Modified posterior vertebral column resection for Kummell disease: Case report. Medicine (Baltimore) 2017;96:e5955.

10. Nishida N, Kato Y, Imajo Y, Kawano S, Taguchi T. Biomechanical study of the spinal cord in thoracic ossification of the posterior longitudinal ligament. J Spinal Cord Med 2011;34:518-522.

11. Matsuyama Y, Sakai Y, Katayama Y, et al. Indirect posterior decompression with corrective fusion for ossification of the posterior longitudinal ligament of the thoracic spine: is it possible to predict the surgical results? Eur Spine $\mathrm{J}$ 2009;18:943-948.

12. Qi Qiang CZ-q, DU Jing-zeng, et al. Surgical treatment of the thoracic and thoracolumbar disc herniations through the posterior far lateral approach[J]. Chin J Orthop 2010. 
13. Kanayama M, Ishida T, Hashimoto T, et al. Role of major spine surgery using Kaneda anterior instrumentation for osteoporotic vertebral collapse. J Spinal Disord Tech 2010;23:53-56.

14. Yang H, Pan J, Wang G. A review of osteoporotic vertebral fracture nonunion management. Spine (Phila Pa 1976) 2014;39:B4-6.

15. Halvorson TL, Kelley LA, Thomas KA, Whitecloud TS, 3rd, Cook SD. Effects of bone mineral density on pedicle screw fixation. Spine (Phila Pa 1976) 1994;19:2415-2420.

16. Bjarke Christensen F, Stender Hansen E, Laursen M, Thomsen K, Bunger CE. Long-term functional outcome of pedicle screw instrumentation as a support for posterolateral spinal fusion: randomized clinical study with a 5-year follow-up. Spine (Phila Pa 1976) 2002;27:1269-1277.

17. Wittenberg RH, Shea M, Swartz DE, Lee KS, White AA, 3rd, Hayes WC. Importance of bone mineral density in instrumented spine fusions. Spine (Phila Pa 1976) 1991;16:647-652.

18. Zhang X, Li S, Zhao X, et al. The mechanism of thoracolumbar burst fracture may be related to the basivertebral foramen. Spine J 2018;18:472-481.

19. Hu PP, Liu XG, Yu M. Cerebrospinal Fluid Leakage after Thoracic Decompression. Chin Med J (Engl) 2016;129:1994-2000.

20. Tan LA, Lopes DK, Fontes RB. Ultrasound-guided posterolateral approach for midline calcified thoracic disc herniation. J Korean Neurosurg Soc 2014;55:383-386.

21. Schar RT, Wilson JR, Ginsberg HJ. Intraoperative Ultrasound-Guided Posterior Cervical Laminectomy for Degenerative Cervical Myelopathy. World Neurosurg 2019;121:62-70.

22. Chongyan W, Zhang X, Li S, et al. Mechanism of formation of intravertebral clefts in osteoporotic vertebral compression fractures: An in vitro biomechanical study. Spine J 2018;18:2297-2301.

23. Takahata M, Ito M, Abumi K, Kotani Y, Sudo H, Minami A. Clinical results and complications of circumferential spinal cord decompression through a single posterior approach for thoracic myelopathy caused by ossification of posterior longitudinal ligament. Spine (Phila Pa 1976) 2008;33:1199-1208.

24. Min JH, Jang JS, Lee SH. Significance of the double- and single-layer signs in the ossification of the posterior longitudinal ligament of the thoracic spine. Neurosurgery 2007;61:118-121; discussion 121-112.

25. Yang DL, Yang SD, Chen Q, Shen Y, Ding WY. The Treatment Evaluation for Osteoporotic Kummell Disease by Modified Posterior Vertebral Column Resection: Minimum of One-Year Follow-Up. Med Sci Monit 2017;23:606-612.

26. Jo DJ, Kim YS, Kim SM, Kim KT, Seo EM. Clinical and radiological outcomes of modified posterior closing wedge osteotomy for the treatment of posttraumatic thoracolumbar kyphosis. J Neurosurg Spine 2015;23:510-517.

27. Imagama S, Ando K, Ito Z, et al. Risk Factors for Ineffectiveness of Posterior Decompression and Dekyphotic Corrective Fusion with Instrumentation for Beak-Type Thoracic Ossification of the Posterior Longitudinal Ligament: A Single Institute Study. Neurosurgery 2017;80:800-808.

28. Kawahara N, Tomita K, Kobayashi T, Abdel-Wanis ME, Murakami H, Akamaru T. Influence of acute shortening on the spinal cord: an experimental study. Spine (Phila Pa 1976) 2005;30:613-620.

29. Kawahara N TK. Circumspinal decompression for thoracic myelopathy due to combined ossification of the posterior longitudinal ligament and yellow ligament. . J Jpn Spine Res Soc 2001;12:450-456.

\section{Figures}



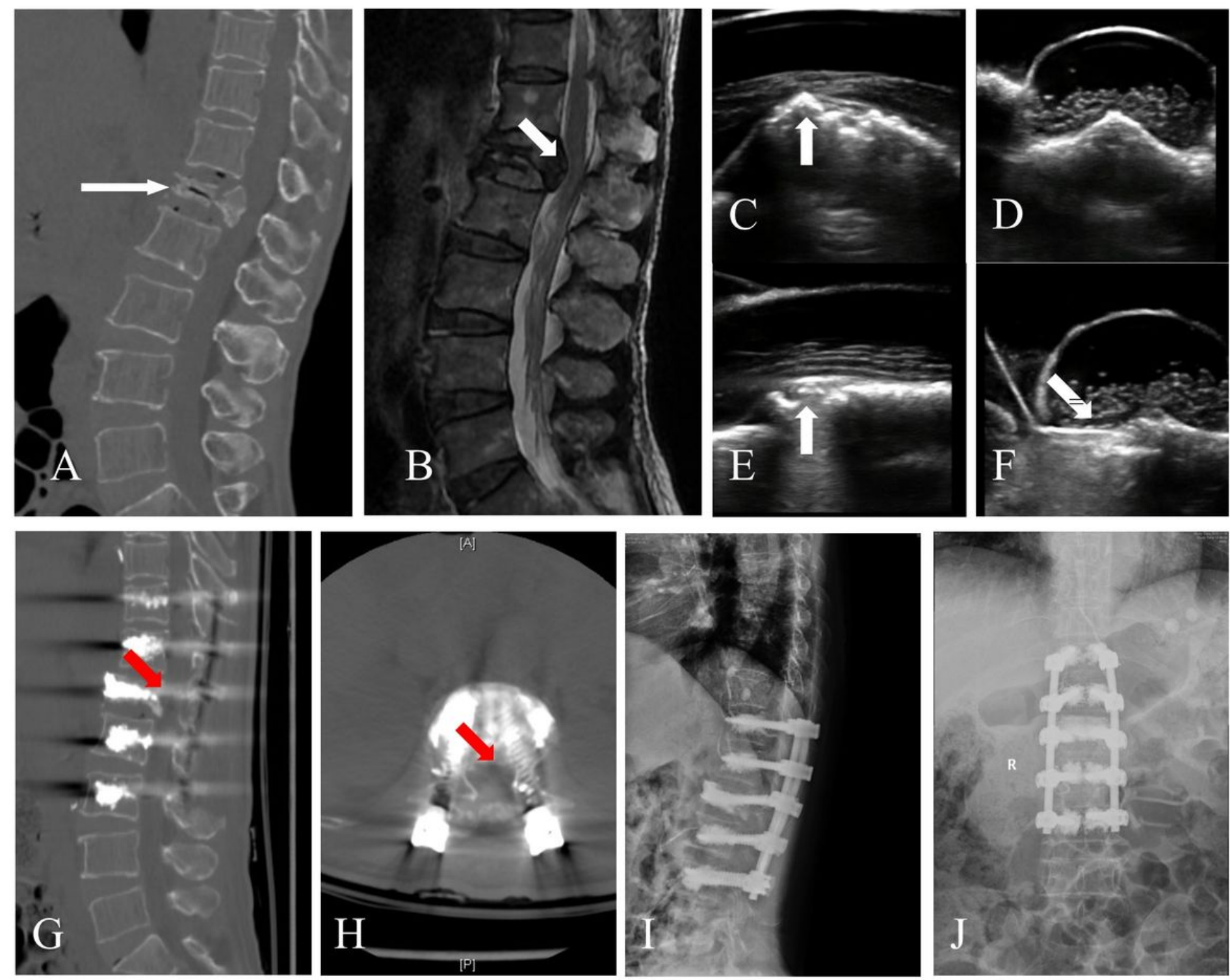

\section{Figure 1}

Radiographic images. A, Reformated CT revealing the L1 vertebral body destructive lesion (arrow). B, the posterior wall of L1 vertebral body displaced into the spinal canal (arrow). C, the real time guidance of ultrasonography: the arrow shows the posterior wall in transverse plane of the spinal canal. D, The sagittal section of the ultrasonography. E, under real-time ultrasonography guidance, the L-shaped compressor (arrow) was put to the ventral side of dural sac through the safe areas. F, the displaced posterior wall (arrow) was compacted and collapsed to the vertebral body. $\mathrm{G}$ and $\mathrm{H}$, the posterior wall (red arrow) was compacted and collapsed to the vertebral body. I and J, the postoperation images. 